\title{
Cooling behaviour of partial thermoremanences induced in multidomain magnetite
}

\author{
Adrian R. Muxworthy ${ }^{1}$ \\ Department of Earth Sciences, University of Oxford, Oxford, UK.
}

\begin{abstract}
The cooling behaviour of partial thermoremanences (pTRM) below the acquisition temperature $\left(T_{2}\right)$ is reported for the first time for well defined, sized, synthetic multidomain magnetite samples produced by hydrothermal recrystallisation. The pTRM cooling behaviour of low-stress magnetite samples, is similar but not identical to that reported for higher stress crushed magnetite samples, that is, it displays a decrease in pTRM on cooling below $\mathrm{T}_{2}$, indicating domain re-organisation. This agrees with recent kinetic theories and domain observations, but strongly disagrees with the classical hysteretic models based on Néel's ideas which assume that the remanence increases with the increase in spontaneous magnetisation. It is demonstrated that the rate of decrease in remanence on cooling is not a viscous effect, and the rate of decrease increases with inducing field dependency. Partial thermoremanence acquired from the Curie temperature $\mathrm{T}_{c}\left(\mathrm{pTRM}_{\mathrm{T}_{2}}^{\mathrm{T}_{\mathrm{c}}}\right)$ is found to be more stable to cooling than pTRM acquired from a temperature $\mathrm{T}_{1}$ which is less than $\mathrm{T}_{c}\left(\mathrm{pTRM}_{\mathrm{T}_{2}}^{\mathrm{T}_{1}}\right)$. It is suggested, that for low-stress samples, pTRM acquired at high temperatures has a wider range of metastable stable states available than pTRM acquired at lower temperatures. The results support the theories that domain wall nucleation is relatively easy during cooling. Differences between the cooling behaviour of the low-stress samples and those previously published for crushed magnetite samples, can be attributed to differences in the dislocations densities and the available metastable domain states.
\end{abstract}

Word count $=7366$

Key words: multidomain; thermoremanent magnetization; magnetite

$\overline{1}$ Now at Institut für Geophysik, Universität München, Theresienstr. 41, 80333 Munich, Germany. E-mail: adrian@geophysik.uni-muenchen.de 


\section{Introduction}

A thermoremanence acquired between the Curie temperature $\left(\mathrm{T}_{c}\right)$ and room temperature is referred to as a TRM, whilst thermoremanence acquired over any other temperature range as a partial thermoremanence or pTRM. According to "classical" hysteretic multidomain (MD) thermoremanence models [1,2], during pTRM acquisition domain walls either block at some temperature $\mathrm{T}_{b}$ or will re-equilibrate to a remanence carrying or demagnetised state when the field is removed at a temperature $T_{2}$, where $T_{2} \leq$ $\mathrm{T}_{b}$. On cooling below $\mathrm{T}_{2}$ it is assumed that pTRM will vary as the ratio of $M_{s}(T) / M_{s}\left(\mathrm{~T}_{2}\right)$ denoted by $m_{s}(T)$, where $M_{s}$ is the spontaneous magnetisation and $T$ the temperature. Because $M_{s}(T)$ increases with decreasing temperature [3], $m_{s}(T) \geq 1$ for all $T \leq \mathrm{T}_{2}$, and so by classical theory, the ratio $\mathrm{pTRM} / m_{s}(T)$ is constant on cooling below $\mathrm{T}_{2}$, however $\mathrm{pTRM} / m_{s}(T)$ has been found experimentally to decrease on cooling below $\mathrm{T}_{2}$ for $\mathrm{MD}$ magnetite [4-8]. Similar results have been found for cooling of isothermal remanences (IRM) induced at $240{ }^{\circ} \mathrm{C}$ in MD magnetite [9]. This decrease in the remanence on cooling indicates that domain re-organisation occurs, which violates the idea of a "blocked" domain structure fundamental to the classical model. It should be noted that viscous decay [10], is not the cause of this decrease in either pTRM or IRM, because no change is observed if the samples are held at a constant temperature for the same length of time[6,9].

In addition to measurement of pTRM below $\mathrm{T}_{2}$, direct observations of domain structure in MD magnetite using optical techniques have found a temperature dependence of domain structure which disagrees with classical domain models [11-13]. The domain structures of synthetic crystals produced by hydrothermal recrystallisation were found to be highly temperature dependent at low temperatures $\left(<100{ }^{\circ} \mathrm{C}\right)$ violating Néel's theories $[11,12]$. In natural crystals the number of domains was found to be relatively constant until $400{ }^{\circ} \mathrm{C}$, but gradually decrease on warming to $555^{\circ} \mathrm{C}$ [13]. Domain structures during TRM acquisition have also been observed, though not for magnetite due to its high $\mathrm{T}_{c}$. Metcalf \& Fuller [14,15] found that on cooling small, MD grains of $\mathrm{Fe}_{2.4} \mathrm{Ti}_{0.6} \mathrm{O}_{4}$ from $\mathrm{T}_{c}$ in the earth's field the number of domains increased with decreasing temperature. In contrast, Halgedahl [16] observed for $\mathrm{Al}_{0.1} \mathrm{Mg}_{0.1} \mathrm{Fe}_{2.2} \mathrm{Ti}_{0.6} \mathrm{O}_{4}$ grains that during cooling the domain structure de-nucleated domain walls. There is clearly an unresolved contradiction between the observations of Metcalf \& Fuller 
$[14,15]$ and Halgedahl [16], however this may be due to problems with domain wall visibility at high temperatures using the Bitter pattern method. Halgedahl [16] noted that domain walls often become visible during cooling, not due to nucleation, but simply due the collection of more colloidal particles around domain walls due to increases in $M_{s}$, a possible reduction in wall width and a reduction in the thermal randomisation of the colloidal particles. The small $\mathrm{Al}$ and $\mathrm{Mg}$ content in Halgedahl's samples reduced $\mathrm{T}_{c}$ from $150{ }^{\circ} \mathrm{C}$ for $\mathrm{Fe}_{2.4} \mathrm{Ti}_{0.6} \mathrm{O}_{4}$ to $75{ }^{\circ} \mathrm{C}$, which improved domain wall visibility near $\mathrm{T}_{c}$, by reducing thermal randomisation processes. Whether of not nucleations or denucleations occur during cooling is still unclear, however, there is strong observational evidence, especially from the work of Halgedahl [16], that disagrees with Néel's domain-wall pinning theory for TRM acquisition. It is worth noting, that it is incorrect to directly apply the results form the titanomagnetite studies, to magnetite, because of the greater importance of magnetostriction in titanomagnetite domain structure. Yet some of the TRM results from titanomagnetite, if correctly, considered can yield important information about the nature of TRM acquisition in magnetite [17].

Recent models of this pTRM cooling behaviour have been less than comprehensive. Incorporating the temperature dependent domain wall nucleation ideas of Moon \& Merrill [18,19], kinematic models has been developed to explain thermoremanence acquisition and its behaviour on cooling below $\mathrm{T}_{2}[6,20]$. McClelland \& Sugiura [6] assumed that domain walls can be regarded as a statistical ensemble, with each element of the ensemble being in one of two possible states: demagnetised or TRM state. During cooling, the domain walls jump randomly between the two states, however in zero-field the demagnetised state is preferred whilst in a field the TRM state is preferred. During TRM acquisition the net contribution from the statistical ensemble is a magnetised state, whilst on cooling below $T_{2}$ the net magnetisation demagnetises giving rise to the observed behaviour. Shcherbakov et al. [20] developed this theory by considering a theoretical micromagnetic approach, i.e., in effect they replaced the statistical ensemble of domain walls used by McClelland \& Sugiura [6], with a statistical ensemble of dipole moments. Unfortunately the model of Shcherbakov et al. [20] makes some simplifications which rather invalidate the model; most importantly the model did not calculate the inter-cellular interaction effect. However the model does predict a decrease in thermoremanence after cool- 
ing in zero-field below $\mathrm{T}_{2}$, allows for the possibility of the irreversibility of pTRM on heating and cooling, and shows the importance of thermal prehistory on remanence acquisition [21].

Attempts to model TRM acquisition using three-dimensional micromagnetic modelling have been limited to SD and vortex structures [22], and have found that nucleation and de-nucleation between these states only occurs at high temperatures. However, the behaviour of these simple domain structures is thought not to be analogous to the behaviour of the larger MD grains [23], rendering comparisons between the model results for SD and vortex structures and the behaviour of larger MD grains speculative.

Clearly there are still unresolved problems in the field of TRM acquisition and the behaviour of pTRM. It is important to understand the mechanisms controlling MD TRM acquisition if, for example, we wish to improve palaeointensity determinations. Previous measurements of pTRM cooling below $\mathrm{T}_{2}$ have been measured on annealed synthetic samples of unknown preparation [5], crushed, sized, natural magnetites [6] and peridotite samples with a near magnetite phase displaying MD characteristics [7]. In this paper, for the first time, the results of cooling pTRM induced in well defined, sized, synthetic MD magnetite samples produced by hydrothermal recrystallisation are reported. Hydrothermal crystals have lower dislocation densities than synthetic samples produced by crushing or by the crystals grown by the glass ceramic method [24], and their magnetic behaviour is closer to that predicted by theoretical models for "ideal" crystals [23]. This makes them perfect for studying fundamental magnetic effects. The effects on cooling behaviour of variations in PTRM inducing field and grain size are investigated.

\section{Sample description and experimental methods}

The four synthetic sample sets $(H(7.5 \mu m)-H(76 \mu m))$ considered in this study, were made by hydrothermal recrystallisation [25]. Grain size distributions and magnetic parameters of the samples are summarised in Table 1. XRD analysis and Mössbauer spectroscopy confirmed that the samples were pure magnetite [26]. The samples have slightly wider grain distributions than the hydrothermal crystals prepared by Heider \& Bryndzia 
[25]. The hydrothermal samples have low values for coercive force $\left(H_{c}\right)$ and low saturation remanence to $\left(M_{r s}\right)$ to saturation magnetization $\left(M_{s}\right)$ ratios suggesting that they have low-dislocation densities, in agreement with previous studies [24]. The samples were dispersed (2-3\% magnetite) in lightlypressed $\mathrm{KBr}$ pellets. Several pellets from each grain size range were prepared.

Thermomagnetic cooling curves were measured using a Princeton Measurements $\mu$-VSM (Vibrating Sample Magnetometer). Two types of pTRM were induced in the samples; Type 1 - samples are cooled from above $\mathrm{T}_{c}$ to $\mathrm{T}_{2}$ in a field, where room temperature, $\mathrm{rtp} \leq \mathrm{T}_{2}<\mathrm{T}_{c}\left(\mathrm{~T}_{c} \downarrow \mathrm{T}_{2}, \mathrm{H}=\mathrm{ON}\right.$; $\mathrm{pTRM}_{\mathrm{T}_{2}}^{\mathrm{T}_{\mathrm{c}}}$. Type 2 - $\mathrm{pTRM}$ acquired by cooling from $\mathrm{T}_{c}$ to a temperature $\mathrm{T}_{1}$ with the field switched off, and from $\mathrm{T}_{1}$ to $\mathrm{T}_{2}$ in a field, where $\mathrm{rtp}<\mathrm{T}_{2}<\mathrm{T}_{1}$ $<\mathrm{T}_{c}\left(\mathrm{~T}_{c} \downarrow \mathrm{T}_{1}, \mathrm{H}=\mathrm{OFF} ; \mathrm{T}_{1} \downarrow \mathrm{T}_{2}, \mathrm{H}=\mathrm{ON} ; \mathrm{pTRM}_{\mathrm{T}_{2}}^{\mathrm{T}_{1}}\right)$. In both cases below $\mathrm{T}_{2}$, the samples were cooled with the field switched off. The $\mu$-VSM allowed the samples to be kept in an inert atmosphere of He during heating which reduced the possibility of oxidation. As an extra precaution against oxidation the pellets were coated in weak-diamagnetic, high-temperature glue (Omega CC High Temperature Cement). Each sample was examined only once.

As the $\mu$-VSM had no shielding from the earth's magnetic field, it was necessary to induce the thermoremanences in fields greater than $\approx 42 \mu \mathrm{T}$ to produce a "step-down" in field when $\mathrm{T}_{2}$ was reached. Most experimental evidence in the literature suggests that TRM intensity measured is linear with applied field up to $200-400 \mu \mathrm{T}[20,27,28]$. Such a TRM is usually referred to as "weak-field" TRM, while TRM induced in higher fields as "high-field" TRM. A field of $\approx 200 \mu \mathrm{T}$ would have been ideal for inducing pTRM, being "weak-field" TRM and with a reasonable step-down in field at $\mathrm{T}_{2}$. Unfortunately, the low sensitivity of the $\mu$-VSM, meant that this was not possible. Initially an inducing field of $5 \mathrm{mT}$, i.e., $5000 \mu \mathrm{T}$ was used, however, a few later samples were induced with pTRM in a field of $500 \mu \mathrm{T}$. TRM induced in fields as high as $5 \mathrm{mT}$ is probably not analogous to thermoremanences induced in the earth's field, and it is debatable that thermoremanences induced in $500 \mu \mathrm{T}$ are analogous either, however, high-field thermoremanences are still of scientific interest, as they provide further contributions to thermoremanence theory even if the results are not directly applicable to naturally occurring thermoremanences. In fact most previous 
studies investigating the decrease in pTRM on cooling below $\mathrm{T}_{2}$, have examined "high-field" thermoremanences, i.e., $H>400 \mu \mathrm{T}$, e.g., Parry [4] and Sugiura [9] both used a field $1 \mathrm{mT}$, whilst McClelland \& Sugiura [6] used a field of $0.84 \mathrm{mT}$. However the observed decrease is not a property confined to high-field thermoremanences, as Markov et al. [5], Sholpo et al. [7] and Shcherbakova et al. [8] have observed the same effect for an inducing field of $100 \mu \mathrm{T}$.

\section{Results}

\subsection{Field dependence of $p$ TRM decay on cooling below $T_{\mathbf{2}}$}

The pTRM inducing field in some of the experiments in this study was considerably higher than those used in all previous studies except for Markov et al. [5]. Because of this difference it was decided to examine the dependency of pTRM cooling behaviour on $H$. Partial thermoremanences acquired over the same temperature range, but with different inducing field $(0.5 \mathrm{mT}$ and $5 \mathrm{mT}$ ), are shown in Figure 1 for Type 1 and Type 2 pTRM. As is standard in these type of diagrams, the increase in the spontaneous magnetisation is compensated for.

The reported decrease in pTRM $/ m_{s}(T)$ on cooling [5-7] is repeated here (Figure 1). However the field dependency of behaviour is opposite to that reported by Markov et al. [5], who found that as $H$ increased the observed demagnetisation in $\mathrm{pTRM}_{300}^{400}$ below $\mathrm{T}_{2}$ decreased. In a similar experiment, Sugiura [9] measured the decrease of IRM on cooling from an acquisition temperature of $240^{\circ} \mathrm{C}$. He also found that as the inducing field increased the relative demagnetisation of IRM decreased. However, the field-dependency results from this study support the theories of McClelland \& Sugiura [6] and Shcherbakov et al. [20], whilst those of Markov et al. [5] and Sugiura [9] disagree. 


\subsection{Viscous decay}

To check that the decrease on cooling (Figure 1) was not a viscous effect, $H(39 \mu \mathrm{m})$ was induced with a $\mathrm{pTRM}_{480}^{\mathrm{T}_{\mathrm{c}}}$ and held at $480{ }^{\circ} \mathrm{C}$ for $\approx 30 \mathrm{~min}-$ utes in zero field (earth's field). The magnetisation was found to remain constant, confirming the theory that demagnetisation on cooling is truly temperature dependent. This corresponds to similar tests reported in the literature $[6,9]$.

\subsection{Test on irreversibility of thermoremanence}

Both measurement of the magnetisation [6-8] and direct observation of the domain structure [11] find that cooling PTRM curves are irreversible to changes in the direction of heating/cooling below $\mathrm{T}_{2}$. A simple check for this phenomenum was done for a selection of samples (Figure 2), where it is seen that hydrothermal recrystallised grains of magnetite behave in a similar manner as crushed grains of magnetite [6]. The size of the decay is similar to that reported by Shcherbakova et al.[8], for a natural sample induced with a pTRM $\mathrm{M}_{300}^{400}$ in a field of $100 \mu \mathrm{T}$.

\subsection{Effect of grain size and pTRM type}

In this section, the dependency of pTRM cooling behaviour on grain size and pTRM type is investigated. For all experiments in this section a field of $5 \mathrm{mT}$ was used to induce the patial thermoremanences. The Type 1 pTRM, i.e., $\mathrm{pTRM}_{\mathrm{T}_{2}}^{\mathrm{T}_{\mathrm{c}}}$, was induced by cooling in field over the temperature range $\mathrm{T}_{c}$ $\rightarrow 520{ }^{\circ} \mathrm{C}$. The results are normalised and the temperature-dependent behaviour of $m_{s}(T)$ compensated for (Figure 3). It is seen in Figure 3, that the demagnetisation observed on cooling $\mathrm{pTRM}_{520}^{\mathrm{T}_{2}}$ decreases with increasing grain size.

Two Type 2 pTRMs, i.e., $\mathrm{pTRM}_{\mathrm{T}_{2}}^{\mathrm{T}_{1}}$, were induced by cooling the samples over the temperature ranges $550{ }^{\circ} \mathrm{C} \rightarrow 450{ }^{\circ} \mathrm{C}$ and $450{ }^{\circ} \mathrm{C} \rightarrow 300^{\circ} \mathrm{C}$, having previously been cooled from $\mathrm{T}_{c}$ in the earth's field. 
For Type 2 pTRM (Figure 4), it is seen that the demagnetisation during cooling pTRM ${ }_{300}^{450}$ below $300{ }^{\circ} \mathrm{C}$ and pTRM $_{450}^{550}$ on cooling below $450{ }^{\circ} \mathrm{C}$, are both relatively independent of grain size. Only pTRM ${ }_{450}^{550}$ induced in sample $H(7.5 \mu \mathrm{m})$ (Figure 4) displays a grain size dependency, and unfortunately no cooling behaviour was measured for sample $H(7.5 \mu \mathrm{m})$ induced with $\mathrm{pTRM}_{300}^{450}$. The rate of demagnetisation on cooling below $\mathrm{T}_{2}$ is greater for $\mathrm{pTRM}_{300}^{450}$ than for $\mathrm{pTRM}_{450}^{550}$, but the amount of decay from $\mathrm{T}_{2}$ to $20{ }^{\circ} \mathrm{C}$ is similar.

Figure 5 depicts both Type 1 and Type 2 pTRM for the $H(76 \mu m)$ sample. Type 2 pTRM i.e., pTRM $_{450}^{550}$ and pTRM $_{300}^{450}$, decrease more rapidly than Type 1 pTRM, i.e., $\mathrm{pTRM}_{520}^{\mathrm{T}_{\mathrm{c}}}$. This suggests that Type $2 \mathrm{pTRM}$ domain structures are more susceptible to domain reconfigurations during cooling below $\mathrm{T}_{2}$, i.e., they are less stable than Type 1 pTRM.

\section{Discussion}

\subsection{Field dependence of $p$ TRM decay}

The field dependency of behaviour depicted in Figure 1, is opposite to that reported by Markov et al. [5]. There is no clear reason for the disagreement, however there are a few possible contributory causes which may account for it. Firstly, the samples in this study were relatively stress-free stoichiometric magnetites, whilst Markov et al. [5] used synthetic magnetites (300$400 \mu \mathrm{m}$ ) of unknown origin. Secondly, the temperature range over which the partial thermoremanences were acquired were different; in this study pTRM was acquired over the range $\mathrm{T}_{c}-520{ }^{\circ} \mathrm{C}$ for Type 1 pTRM and $450-$ $300{ }^{\circ} \mathrm{C}$ for Type 2 pTRM, whereas Markov et al. [5] induced their pTRM (Type 2 only) over the temperature range $400-300{ }^{\circ} \mathrm{C}$. However it is unlikely that pTRMs acquired over $450-300{ }^{\circ} \mathrm{C}$ and $400-300{ }^{\circ} \mathrm{C}$ should vary significantly in there response to applied field. Lastly, differences in initial domain state could play a significant role. In this study for Type 2 pTRM, the samples were cooled from $\mathrm{T}_{c}$ in zero-field (in fact the earth's field). This domain state is commonly referred to as the absolute zero state (AZS) [20]. The experiments of Markov et al. [5] were carried out before the importance of thermal history had been truly clarified [21]. Because of this Markov et al. 
[5] have not commented on the thermal histories of their samples, only that they were induced with $\mathrm{pTRM}_{300}^{400}$. It is unknown if differences in thermal histories affect the field dependency of pTRM cooling behaviour. It should be noted in support of the results of Markov et al. [5], however, that the similar field dependency behaviour observed by Sugiura [9], was for IRMs induced in AZS MD grains.

It was noted above that the $\mathrm{pTRM}_{300}^{450}$ was not induced in hydrothermal samples in a true AZS, because they had been cooled from $\mathrm{T}_{c}$ in the earth's field. However, pTRM $_{520}^{T_{c}}$ displayed a similar field dependency (Figure 1) as $\mathrm{pTRM}_{300}^{450}$ which suggests that the error in the AZS of $\mathrm{pTRM}_{300}^{450}$ was not significant.

\subsection{Effect of grain size and pTRM type}

On cooling Type 1 pTRM the observed demagnetisation (Figure 3) decreases with increasing grain size, contrary to previous experimental observations which found an increase [6,9]. However there are differences in the detail; in this study thermoremanences were induced in a larger field $(H=5 \mathrm{mT})$ than either McClelland \& Sugiura [6, $H=0.84 \mathrm{mT}]$ or Sugiura [9, $H=1 \mathrm{mT}]$. Secondly McClelland \& Sugiura [6] and Sugiura [9] both used crushed magnetites, whereas the samples in this study were relatively stress-free.

It is suggested here, that the increased inducing field can partially explain the discrepancy between the literature and the results in this study. The larger inducing field would be expected to form domain configurations with fewer domains than those induced in the smaller field by McClelland \& Sugiura [6]. On switching off the field at $\mathrm{T}_{2}$, the domain structure of MD grains partially reverses, the "reversible induced moment" (RIM) of McClelland \& Sugiura [6], i.e., the high self-demagnetising energy causes unblocked domain walls to demagnetise until pinned on a dislocation. Because hydrothermal crystals have lower internal stresses and less pinning sites than crushed crystals, hydrothermal samples are expected to have larger RIM than the crushed rocks of McClelland \& Sugiura [6]. In this study over $90 \%$ of the moment was reversible on switching off the $5 \mathrm{mT}$ field (Table 2), compared to McClelland \& Sugiura [6] who found RIM values in their crushed samples in the range $50-95 \%$ depending on the selected 
pTRM temperature range for an inducing field of $0.84 \mathrm{mT}$. As the inducing field in this study was higher than that used by McClelland \& Sugiura [6] it is difficult to draw direct comparisons.

As expected the size of the RIM was dependent on the inducing field (Table 2). The smaller crystals, e.g., $H(7.5 \mu \mathrm{m})$, have smaller RIM than the larger crystals, e.g., H(76 $\mu \mathrm{m})$, (Table 2$)$, in agreement with the results of McClelland \& Sugiura [6]. There are two reasons for this; firstly the smaller grains have fewer domain walls to reverse, and secondly there is a relatively larger interaction between the domain walls and the crystals surfaces. It is even possible that small crystals are in a SD-like state after cooling from $\mathrm{T}_{c}$ in a field of $5 \mathrm{mT}[28,29]$. On cooling below $\mathrm{T}_{2}$, the smaller crystals are expected to be in relatively unstable high-magnetisation states, making them susceptible to domain reconfigurations. Due to the low-number of domains, nucleation of even one wall contributes significantly to the domain structure. In larger grains with many more domains, the nucleation of one or two walls is less significant. On removal of the field at $\mathrm{T}_{2}$, the crushed samples of McClelland \& Sugiura [6] have larger remanence per grain than hydrothermal crystals, because of the increased pinning site density, i.e., domain walls are trapped in states further from the demagnetised state. The demagnetisation process which occurs on cooling, is expected to increase as the selfdemagnetising energy domain structure increases, i.e., the process is more pronounced in crushed grains than for hydrothermal grains. Increased dislocation density aids the ability of domain walls to nucleate/denucleate [23].

Type 2 pTRM is relatively independent of grain size (Figure 4), unlike Type 1 pTRM which displays a strong grain size dependency (Figure 3 ). This may reflect a narrower range of possible domain configurations open to Type 2 pTRM.

In comparing Type 1 and Type 2 pTRM (Figure 5), it is immediately obvious that Type 1 pTRM is more stable on cooling than Type 2. This is in disagreement with McClelland \& Sugiura [6], but in agreement with Sholpo et al. [7]. This difference may be due to the nature of the samples, as the former used crushed samples, whereas the later used natural samples.

A pTRM acquired at a higher temperature range, i.e., $\mathrm{pTRM}_{\mathrm{T}_{2}}^{\mathrm{T}_{\mathrm{c}}}$ will form domain structures with higher self-demagnetising energies, than a pTRM ac- 
quired at lower temperatures. It is postulated, that increases in the dislocation density will also cause domain structures with higher self-demagnetising energies to be formed, i.e., grains with high-dislocation densities are expected to have less domains. This is supported by domain observations, on two sets of natural magnetites, where it was found that the samples displaying higher coercivity of remanence (often considered to be related to internal stress), also displayed on average less domains at higher temperatures [13]. Therefore a PTRM acquired in a crushed grain at high temperatures is expected to have less domains and consequently a very high self-demagnetising energy. During cooling domain structures with higher self-demagnetising energies are more likely to be demagnetised. Assuming that the results from micromagnetic calculations for pseudo-single domain grains are applicable to larger grains, then it is postulated that for MD grains that the number of accessable metastable LEM structures decreases with temperature [30], even though the number of metastable LEM states increases [31]. Samples with low-internal stress will have on average a smaller remanence per grain, however, as the energy barriers between the various LEM states are likely to be smaller on average than in stressed samples the domain configuration will have a wider range of available metastable LEM states. In low-stress samples with smaller self-demagnetising energies, LEM structures acquired at high temperatures are relatively stable to the self-demagnetising energy. Consequently pTRM acquired in lowstress samples is more stable if acquired at higher temperatures. Unstable domain structure will display the largest demagnetisation on cooling, i.e., $\mathrm{pTRM}_{\mathrm{T}_{2}}^{\mathrm{T}_{\mathrm{c}}}$ is less stable than $\mathrm{pTRM}_{\mathrm{T}_{2}}^{\mathrm{T}_{1}}$ for crushed samples, and vice versa for natural and hydrothermal samples.

\subsection{Implications of high inducing and residual field}

The inducing field and residual field used in this paper were higher than those used in previous studies. Here the implications of these high fields are discussed.

The two inducing fields used, i.e., $0.5 \mathrm{mT}$ and $5 \mathrm{mT}$, are both thought to have produced "high-field" thermoremanences, i.e., where the TRM acquisition no longer displays a linear relationship with inducing field intensity. The $0.5 \mathrm{mT}$ inducing field is believed to be just above the break in 
linear behaviour (0.2-0.4 mT) [28]. It is likely that high-field thermoremanences are relatively less stable than weak-field thermoremanences, however, once a wall is pinned or blocked, according to Néel [1] the energy barrier will increase on cooling and the wall will remain blocked, regardless of whether the initial remanence is high- or weak-field thermoremanence or of some other origin, e.g., isothermoremanence, that is, the remanence should increase as $M_{s}$ on cooling. The behaviour of high-field remanences in this study disagrees with the theory of Néel [1]. On comparison to previous studies, the $5 \mathrm{mT}$ inducing field was a little higher than those used in other studies, but the $0.5 \mathrm{mT}$ field was less than that used in several previous high-field thermoremanence studies [4,6,9]. All high-field pTRMs displayed a similar decrease in magnetisation on cooling. This effect has also been observed for weak-field pTRM $[5,7,8]$. It appears, therefore, that the cooling behaviour is independent of the type of inducing field whether it be low or high field thermoremanence. However, whether results from highfield thermoremanences are directly applicable to low-field thermoremanence studies is debatable, but the implications and interpretations still have relevance to natural thermoremanence studies.

As the measurements were made in the earth's field, and not in an absolute zero field as is desired, then this leads to the question: is it possible that the high-"zero field" approximation is the cause of demagnetisation on cooling below $\mathrm{T}_{2}$ and that it is not a genuine MD effect independent of field? There are several arguments which suggest that the behaviour is an MD effect. Firstly, on switching off the inducing field, the field is stepped down from $5 \mathrm{mT}$ to the earth's field orientated in a different direction. Domains walls which pin at this temperature, are pinning in the presence of the earth's magnetic field. So as the earth's magnetic field is constant during cooling, the energy barriers and conditions due to the residual field should not change. This effectively reduces or increases the energy barriers compared to an ideal zero field situation, but does not change the energy barrier's behaviour on cooling. On cooling the energy barriers should increase in an identical manner to that of the ideal zero-field case, and the behaviour should be the same, i.e., it is the step down in field which is important.

Another argument in support of the idea that it is genuine MD effect is found by considering the viscous magnetisation experiment discussed above (section 3.2). If the earth's field was the cause of the demagnetisation, then 
the effect of the earth's field would be greater at higher temperatures. However, when the temperature was held constant as $480{ }^{\circ} \mathrm{C}$, as discussed previously, the magnetisation was found to remain constant. This suggests that the earth's magnetic field did not cause significant demagnetisation of the remanence and that the decrease in magnetisation is a genuine MD temperature effect. Lastly, the same decrease on cooling has been seen in previous studies where the residual field was lower, suggesting that the earth's field was not the cause of the demagnetisation. In the limited number of previous studies of this type, the "zero-field" approximation was usually less than $\approx 10 \mu \mathrm{T}[4-8]$.

However, it is also necessary to consider the induced moment due to the earth's magnetic field, i.e., the RIM in the earth's field. Unfortunately this was not directly measured as a function of temperature in this study. However, McClelland \& Sugiura [6] did measure it as a function of temperature for a field of $0.84 \mathrm{mT}$. They found that it decreased with temperature, but not as rapidly as the decrease in pTRM due to cooling. Importantly they found that RIM and the remanence were effectively independent of each other. If the RIM due to the earth's magnetic field in this study was significant, then its effects would have been observed in two experiments. As the RIM due to the earth's field is proportionally largerer in PTRM induced in a field of $0.5 \mathrm{mT}$ compared to a field of $5.0 \mathrm{mT}$, then if the cooling behaviour of the RIM was the cause of the observed cooling decrease in magnetic moment, its effect would have been greater in the experiment where the sample was induced in a field of $0.5 \mathrm{mT}$ and not $5.0 \mathrm{mT}$. However, in Figure 1 it is seen that the $5 \mathrm{mT}$ curves decrease more rapidly, suggesting that a RIM contribution is not significant to the behaviour. Secondly, as McClelland \& Sugiura [6] found that RIM increased slowly with temperature up to $\approx 520^{\circ} \mathrm{C}$, then if the RIM contribution was the cause of the cooling behaviour, then in Figure 2 the warming curve would be expected to increase and not decrease as was observed. It would appear, therefore, that the MD effect was genuine, however, it should also be realised the behaviour of RIM may have also contributed slightly to the observed behaviour, however this is also true for all other studies where there was a residual field. 


\section{Conclusions}

The results presented in this paper agree with those previously published, in that they show a decrease in $\mathrm{pTRM} / m_{s}$ on cooling below $\mathrm{T}_{2}$, indicating domain re-organisation. This agrees with the kinetic theories $[6,20]$ and domain observations [11,12], and strongly disagrees with the classical hysteretic models $[1,2]$ which assume that the domain structure is "blocked".

There are several fundamental points which arise from the results in this study; firstly that the dependency of inducing field on pTRM cooling behaviour agrees with the theory $[6,20]$ contrary to previous reports [5], secondly pTRM $\mathrm{T}_{\mathrm{T}_{2}}^{\mathrm{T}_{\mathrm{c}}}$ appears to more stable for "low-stress" samples than $\mathrm{pTRM}_{\mathrm{T}_{2}}^{\mathrm{T}_{1}}$ on cooling below $\mathrm{T}_{2}$, and thirdly the level of internal stress is thought to strongly effect the cooling behaviour of pTRM.

The results support the theories of McClelland \& Sugiura [6] and Shcherbakov et al. [20], which are, however, too abstract to be quantitative. These theories do not incorporate variations in dislocation density, which is the probable cause for the difference between the results of this study and those of McClelland \& Sugiura [6]. McClelland \& Sugiura [6] and Shcherbakov et al. [20] suggest that domain wall nucleation is a relatively easy process, but it has been suggested elsewhere that it is not $[17,23]$. However, the arguments against easy domain wall nucleation in magnetite seem flawed. For example, Dunlop \& Özdemir [23] applied the results of Halgedahl [16] to magnetite. Halgedahl [16] explained the behaviour of TRM acquisition in $\mathrm{Al}_{0.1} \mathrm{Mg}_{0.1} \mathrm{Fe}_{2.2} \mathrm{Ti}_{0.6} \mathrm{O}_{4}$ by suggesting that domain nucleation is relatively difficult during cooling, i.e., nucleation failure is more common. However, it is inappropriate to directly apply the results for $\mathrm{Al}_{0.1} \mathrm{Mg}_{0.1} \mathrm{Fe}_{2.2} \mathrm{Ti}_{0.6} \mathrm{O}_{4}$ to magnetite due to the relative importance of the magnetostriction in Ti-rich minerals. From the results in this study, it appears that nucleation is relatively easy, with increased dislocation density facilitating domain wall nucleation in larger grains.

The results in this paper also support the postulation by Heider [12] that the demagnetisation processes on cooling are continuous rather than discrete as suggested by the simplified model of McClelland \& Sugiura [6].

It is planned in a future study to investigate the effect of stress on this phe- 
nomena in more detail, using a suite of samples where the degree of internal stress is more readily controlled, and the residual field is significantly lower.

\section{Acknowledgements}

The work was carried out during the tenure of a NERC research studentship. I would like to thank E. McClelland for her insightful comments and fruitful discussions, and W. Williams, S. Halgedahl and V. Shcherbakov for their

helpful reviews. The measurements were carried out at the the Institute for Rock Magnetism, University of Minnesota.

\section{References}

[1] L. Néel, Some theoretical aspects of rock magnetism, Adv. Phys., 4, 191-242, 1955.

[2] D. J. Dunlop and S. Xu, Theory of partial thermoremanence in multidomain grains: 1 . repeated identical barriers to wall motion (single coercivity), J. Geophys. Res., 99(B5), 9005-9023, 1994.

[3] R. Pauthenet and L. Bochirol, Aimantation spontanée des ferrites, J. Phys. Rad., 12, 249-251, 1951.

[4] L. G. Parry, Magnetization of multidomain particles of magnetite, Phys. Earth Planet. Inter., 19, 12-30, 1979.

[5] G. P. Markov, V. P. Shcherbakov, A. S. Bol'shakov and Yu. K. Vinogradov, On the temperature dependence of the partial thermoremanent magnetization of multidomain grains, Izv., Acad. of Sci., USSR, Phys. Solid Earth, 19(8), 625-630, 1983.

[6] E. McClelland and N. Sugiura, A kinematic model of trm acquisition in multidomain magnetite, Earth Planet. Sci. Lett., 46, 9-23, 1987. 
[7] L. Ye. Sholpo, V. A. Ivanov and G. P. Borisova, Thermomagnetic effects of reorganization of domain structure, Izv., Acad. of Sci., USSR, Phys. Solid Earth, 27(7), 617-623, 1991.

[8] V. V. Shcherbakova, V. P. Shcherbakov and F. Heider, Properties of partial thermoremanent magnetization in pseudosingle domain and multidomain magnetite grains, J. Geophys. Res., 105, 767-781, 2000.

[9] N. Sugiura, A new model for the acquisition of thermoremanence by multidomain magnetite, Can. J. Earth Sci., 18, 789-794, 1981.

[10] D. J. Dunlop, Theory of magnetic viscosity of lunar and terrestrial rocks, Rev. Geophys. Space Phys., 11, 851-901, 1973.

[11] F. Heider, S. L. Halgedahl and D. J. Dunlop, Temperature dependence of magnetic domains in magnetite crystals, Geophys. Res. Lett., 15, 499-502, 1988.

[12] F. Heider, Temperature dependence of domain structure in natural magnetite and its significance for multi-domain trm models, Earth Planet. Sci. Lett., 65, 54-61, 1990.

[13] A. Ambatiello, K. Fabian and V. Hoffmann, Magnetic domain structure of multidomain magnetite as a function of temperature: observation by Kerr microscopy, Phys. Earth Planet. Inter., 112, 55-80, 1999.

[14] M. Metcalf and M. Fuller, Domain observations of titanomagnetites from room temperature to Curie point and the nature of thermoremanent magnetism in fine particles, Nature, 321, 847-849, 1986.

[15] M. Metcalf and M. Fuller, Domain observations of titanomagnetites during hysteresis at elevated temperatures and thermal cycling, Phys. Earth Planet. Inter., 46, 120-126, 1987.

[16] S. L. Halgedahl, Magnetic domain patterns observed on synthetic Ti-rich titanomagnetite as a function of temperature and in states of thermoremanent magnetization, J. Geophys. Res., 96(B3), 3943-3972, 1991.

[17] J. Ye and R. T. Merrill, Use of renormalization group theory to explain the large variation of domain states observed in titanomagnetites and implications for paleomagnetism, J. Geophys. Res., 100(B9), 17899-17907, 1995. 
[18] T. Moon and R. T. Merrill, The magnetic moments of non-uniformly magnetized grains, Phys. Earth Planet. Inter., 34, 186-194, 1984.

[19] T. Moon and R. T. Merrill, Nucleation theory and domain states in multidomain magnetic material, Phys. Earth Planet. Inter., 37, 214-222, 1985.

[20] V. P. Shcherbakov, E. McClelland and V. V. Shcherbakova, A model of multidomain thermoremanent magnetisation incorporating temperaturevariable domain structure, J. Geophys. Res., 98(B4), 6201-6216, 1993.

[21] Yu. K. Vinogradov and G. P. Markov, On the effect of low temperature heating on the domain state of multidomain magnetite, in: Investigations in rock magnetism and palaeomagnetism (in Russian), pp. 31-39, Institute of Physics of the Earth, Moscow, 1989.

[22] M. Winklhofer, K. Fabian and F. Heider, Magnetic blocking temperature of magnetite calculated with a three-dimensional micromagnetic model, J. Geophys. Res., 102(B10), 22695-22709, 1997.

[23] D. J. Dunlop and Ö. Özdemir, Rock Magnetism: Fundamentals and Frontiers, Cambridge University Press, 1997.

[24] F. Heider, D. J. Dunlop and N. Sugiura, Magnetic properties of hydrothermally recrystallised magnetite crystals, Science, 236, 1287-1290, 1988.

[25] F. Heider and L. T. Bryndzia, Hydrothermal growth of magnetite crystals (1 $\mu \mathrm{m}$ to $1 \mathrm{~mm})$, J. Crystal Growth, 84, 50-56, 1987.

[26] A. R. Muxworthy, Stability of magnetic remanence in multidomain magnetite, D.Phil. thesis, University of Oxford, 1998.

[27] R. Day, TRM and its variation with grain size, J. Geomag. \& Geoelec., 29, 233$265,1977$.

[28] D. J. Dunlop and K. S. Argyle, Thermoremanence, anhysteretic remanence and susceptibility of submicron magnetites: Nonlinear field dependence and variation with grain size, J. Geophys. Res., 102(B9), 20199-20210, 1997.

[29] J. R. Boyd, M. Fuller and S. Halgedahl, Domain wall nucleation as a controlling factor in the behaviour of fine magnetic particles in rock, Geophys. Res. Lett., 11(3), 193-196, 1984. 
[30] D. J. Dunlop, A. J. Newell and R. J. Enkin, Transdomain thermoremanent magnetization, J. Geophys. Res., 99(B10), 19741-19755, 1994.

[31] T. S. Moon, Domain states in fine particle $\mathrm{Fe}_{3} \mathrm{O}_{4}$ and titanomagnetite, J. Geophys. Res., 96(B6), 9909-9923, 1991. 
Table 1

Summary of mean grain size, standard deviation $\sigma$, and hysteresis parameters $H_{c}$, $H_{c r}$ (remanent coercive force) and the ratio of $M_{r s}$ to $M_{s}$ at room temperature, for the samples considered in this study.

\begin{tabular}{ccccccc}
\hline sample & size & $\pm \sigma$ & $H_{c}$ & $H_{c r}$ & \\
name & $(\mu \mathrm{m})$ & $(\mu \mathrm{m})$ & $(\mathrm{mT})$ & $(\mathrm{mT})$ & $M_{r s} / M_{s}$ \\
\hline$H(7.5 \mu \mathrm{m})$ & 7.5 & 3.0 & 2.4 & 18.4 & 0.018 \\
$H(39 \mu \mathrm{m})$ & 39 & 9 & 1.5 & 24.7 & 0.010 \\
$H(59 \mu \mathrm{m})$ & 59 & 16 & 1.36 & 15.8 & 0.008 \\
$H(76 \mu \mathrm{m})$ & 76 & 25 & 0.96 & 19.9 & 0.006 \\
\hline
\end{tabular}


Table 2

Reversible induced moment (RIM) for $\mathrm{pTRM}_{520}^{\mathrm{T}_{\mathrm{c}}}$ for different applied fields, as a percentage of the total magnetic moment at $520^{\circ} \mathrm{C}$.

\begin{tabular}{ccc}
\hline sample & field $(\mathrm{mT})$ & RIM \\
\hline$H(7.5 \mu \mathrm{m})$ & 5 & $91.7 \%$ \\
$H(39 \mu \mathrm{m})$ & 5 & $96.9 \%$ \\
$H(59 \mu \mathrm{m})$ & 5 & $97.5 \%$ \\
$H(76 \mu \mathrm{m})$ & 5 & $97.6 \%$ \\
$H(76 \mu \mathrm{m})$ & 0.5 & $93.3 \%$ \\
\hline
\end{tabular}


Fig. 1. Cooling behaviour of normalised Type $1\left(\operatorname{pTRM}_{520}^{\mathrm{Tc}_{\mathrm{c}}} / m_{s}(T)\right)$ and Type 2 $\left(\mathrm{pTRM}_{300}^{450} / m_{s}(T)\right)$ for hydrothermal sample $H(76 \mu \mathrm{m})$, with variable inducing field. Measurement was made in the earth's magnetic field.

Fig. 2. Typical thermomagnetic curve depicted the irreversibility of pTRM to changes in heating/cooling direction. pTRM $_{300}^{450}(H=5 \mathrm{mT})$ induced in hydrothermal magnetite sample $H(76 \mu \mathrm{m})$. Measurement was made in the earth's magnetic field.

Fig. 3. Behaviour of normalised $\mathrm{pTRM}_{520}^{\mathrm{T}_{\mathrm{c}}}$ on cooling below $520{ }^{\circ} \mathrm{C}$ in the earth's field. PTRM $_{520}^{\mathrm{T}_{\mathrm{c}}}$ was induced in the hydrothermal samples $H(7.5 \mu \mathrm{m}), H(39 \mu \mathrm{m})$, $H(59 \mu \mathrm{m})$ and $H(76 \mu \mathrm{m})$ using a field of $5 \mathrm{mT}$. Measurement was made in the earth's magnetic field.

Fig. 4. Change of normalised pTRM $/ m_{s}(T)$ on cooling in the earth's field for two different $\mathrm{pTRM}_{\mathrm{T}_{2}}^{\mathrm{T}_{1}}$ induced in hydrothermal samples using a field of $5 \mathrm{mT}$; $\mathrm{pTRM}_{450}^{550}$ induced in samples $H(7.5 \mu m), H(39 \mu m), H(59 \mu m)$ and $H(76 \mu m)$, and pTRM $_{300}^{450}$ induced in samples $H(39 \mu m), H(59 \mu m)$ and $H(76 \mu m)$. Measurement was made in the earth's magnetic field.

Fig. 5. Change of normalised pTRM $/ m_{s}(T)$ on cooling below $\mathrm{T}_{2}$ in the earth's field for sample $H(76 \mu m)$ induced with Type 1 pTRM (pTRM $\left.{ }_{520}^{T_{c}}\right)$ and Type 2 pTRM $\left(\mathrm{pTRM}_{450}^{550}\right.$ and $\left.\mathrm{pTRM}_{300}^{450}\right)$. An inducing field of $5 \mathrm{mT}$ was used. Measurement was made in the earth's magnetic field. 


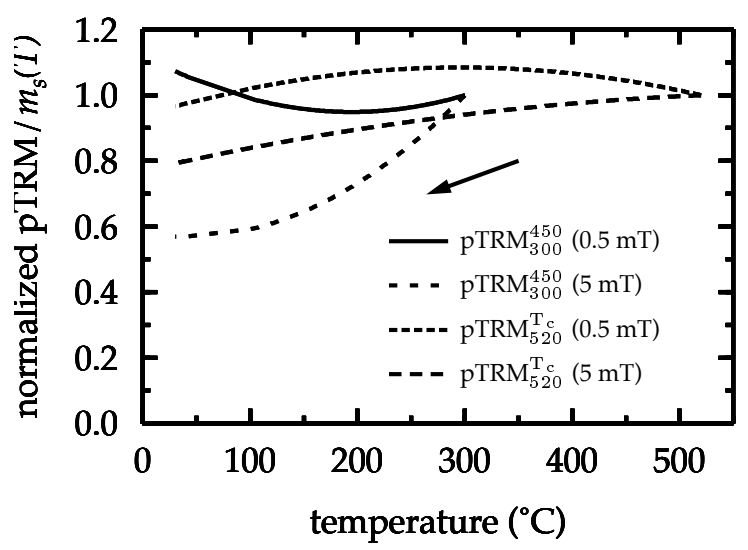




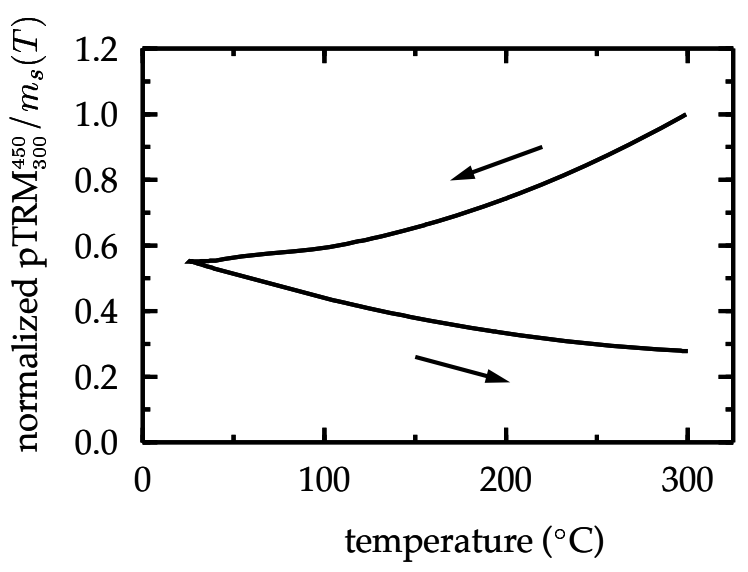




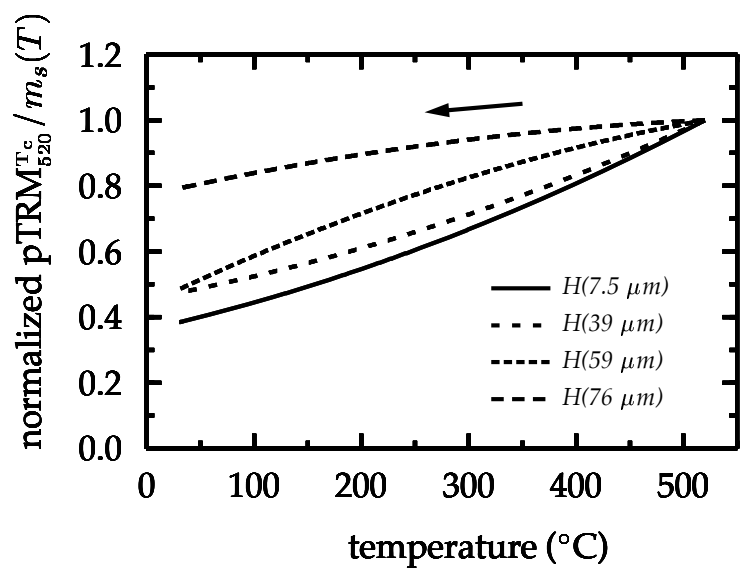




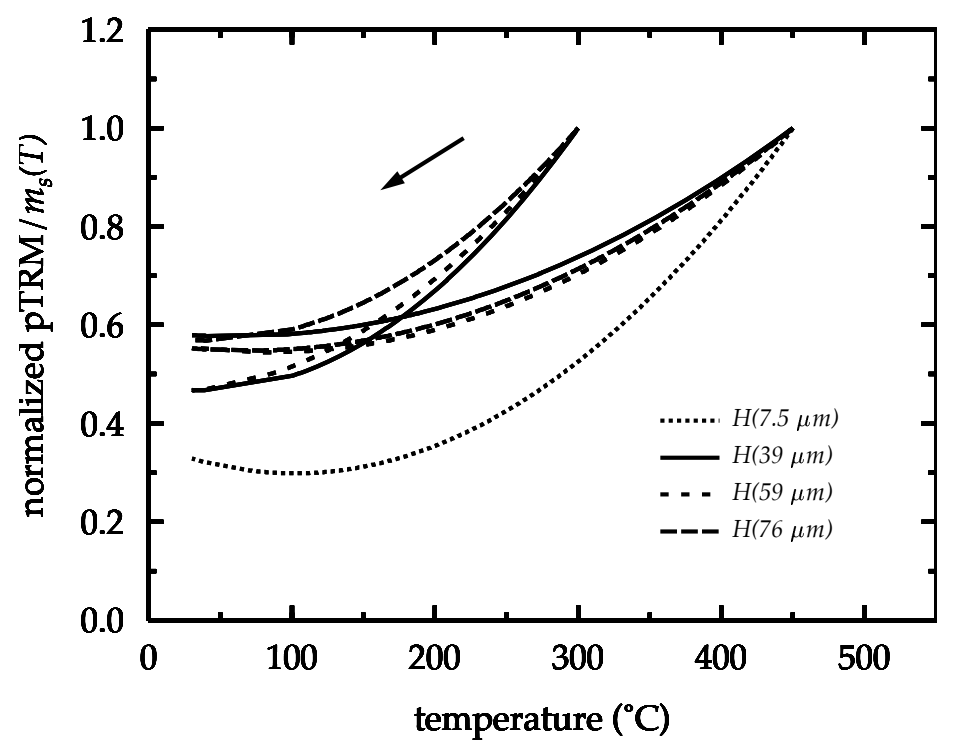




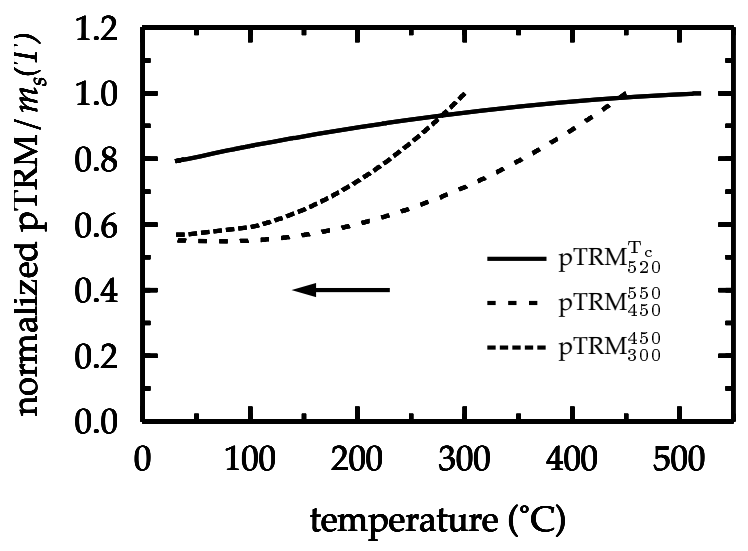

